\title{
Radiative-transfer models for explosions from rotating and non-rotating single WC stars Implications for SN 1998bw and LGRB/SNe
}

\author{
Luc Dessart ${ }^{1}$, D. John Hillier ${ }^{2}$, Sung-Chul Yoon ${ }^{3}$, Roni Waldman ${ }^{4}$, and Eli Livne ${ }^{4}$ \\ 1 Unidad Mixta Internacional Franco-Chilena de Astronomía (CNRS UMI 3386), Departamento de Astronomía, \\ Universidad de Chile, Camino El Observatorio 1515, Las Condes, Santiago, Chile \\ e-mail: Luc.Dessart@oca.eu \\ 2 Department of Physics and Astronomy \& Pittsburgh Particle Physics, Astrophysics, and Cosmology Center (PITT PACC), \\ University of Pittsburgh, 3941 O'Hara Street, Pittsburgh, PA 15260, USA \\ 3 Department of Physics and Astronomy, Seoul National University, Gwanak-ro 1, Gwanak-gu, 151-742 Seoul, Republic of Korea \\ 4 Racah Institute of Physics, The Hebrew University, 91904 Jerusalem, Israel
}

Received 27 March 2017 / Accepted 30 April 2017

\begin{abstract}
Using 1D, non-local thermodynamic equilibrium and time-dependent radiative transfer simulations, we study the ejecta properties required to match the early- and late-time photometric and spectroscopic properties of supernovae ( $\mathrm{SNe}$ ) associated with long-duration $\gamma$-ray bursts (LGRBs). Matching the short rise time, narrow light curve peak and extremely broad spectral lines of SN 1998bw requires a model with $\lesssim 3 M_{\odot}$ ejecta but a high explosion energy of a few $10^{52} \mathrm{erg}$ and $0.5 M_{\odot}$ of ${ }^{56} \mathrm{Ni}$. The relatively high luminosity, presence of narrow spectral lines of intermediate mass elements, and low ionisation at the nebular stage, however, are matched with a more standard C-rich Wolf-Rayet (WR) star explosion, an ejecta of $\gtrsim 10 M_{\odot}$, an explosion energy $\gtrsim 10^{51} \mathrm{erg}$, and only $0.1 M_{\odot}$ of ${ }^{56} \mathrm{Ni}$. As the two models are mutually exclusive, the breaking of spherical symmetry is essential to match the early- and late-time photometric and spectroscopic properties of SN 1998bw. This conclusion confirms the notion that the ejecta of SN 1998bw is highly aspherical on large scales. More generally, with asphericity, the energetics and ${ }^{56} \mathrm{Ni}$ masses of LGRB/SNe are reduced and their ejecta masses are increased, favouring a massive fast-rotating Wolf-Rayet star progenitor. Contrary to persisting claims in favour of the proto-magnetar model for LGRB/SNe, such progenitor/ejecta properties are compatible with collapsar formation. Ejecta properties of LGRB/SNe inferred from 1D radiative-transfer modelling are fundamentally flawed.
\end{abstract}

Key words. radiative transfer - hydrodynamics - supernovae: general

\section{Introduction}

Although some uncertainty remains on the properties of type Ibc supernovae $(\mathrm{SNe})$ and their progenitors, the general consensus is that they arise from H-deficient stars with Wolf-Rayet (WR) like abundances and with a final mass at core collapse of $\sim 5 M_{\odot}$. Their ejecta have a kinetic energy of $\sim 10^{51} \mathrm{erg}$, which is typical of what is inferred for H-rich core-collapse $\mathrm{SNe}$ (Ensman \& Woosley 1988; Drout et al. 2011; Taddia et al. 2015; Prentice et al. 2016). Owing to their low mass most of these objects are thought to arise in binary systems in which one, or both stars, have undergone mass transfer (Podsiadlowski et al. 1992; Eldridge et al. 2008). The fate of single WR stars, which have a large final mass at collapse (Crowther 2007), is however unclear (Dessart 2015). Type Ib and Ic SNe are distinguished by the presence/absence of He I lines in optical spectra, perhaps because type Ic progenitors have a smaller mass of $\mathrm{He}$ and a larger $\mathrm{CO}$ core (see e.g. Georgy et al. 2009; Dessart et al. 2011; Liu et al. 2016).

The broad-line (BL) type Ic $\mathrm{SNe}$ (hereafter $\mathrm{SNe}$ Ic-BL) are observationally distinct from normal type Ibc SNe. Their spectral lines are so broad at early times that line identifications are compromised and the velocity at maximum absorption is uncertain. Nonetheless, their optical spectra exhibit three to four smooth ripples that imply a large expansion rate with a photospheric velocity that may approach $30000 \mathrm{~km} \mathrm{~s}^{-1}$ prior to maximum (for a recent discussion, see Modjaz et al. 2016). Their large expansion rate, association in some cases with longduration $\gamma$-ray bursts (LGRB) ${ }^{1}$, and large luminosities suggest that a subset of core-collapse $\mathrm{SNe}$ produce much larger explosion energies and a much greater mass of ${ }^{56} \mathrm{Ni}$ (see e.g. Iwamoto et al. 1998; Woosley et al. 1999).

One notorious example presenting these extraordinary properties is SN 1998bw, which is an LGRB/SN (Galama et al. 1998). Using 1D radiation hydrodynamics simulations and OPAL opacities, Woosley et al. (1999) find that a $\sim 4.77 M_{\odot}$ CO-rich ejecta with $0.49 M_{\odot}$ of ${ }^{56} \mathrm{Ni}$ and a kinetic energy of $2.8 \times 10^{52} \mathrm{erg}$ yields a satisfactory match to the light curve of SN 1998bw prior to $\sim 100 \mathrm{~d}$. This model corresponds to a $6.55 M_{\odot}$ C-rich WR progenitor star leaving behind a $1.78 M_{\odot}$ neutron star remnant. These authors find that higher mass models yield a rise time that is too long. Using a more simplified light curve model with a prescribed opacity, Iwamoto et al. (1998) argue for $\mathrm{a} \gtrsim 10 M_{\odot}$ ejecta with $2-5 \times 10^{52} \mathrm{erg}$. This is more than twice the ejecta mass of Woosley et al. (1999). An alternative

\footnotetext{
1 All LGRB/SNe are broad-lined type Ic SNe but not all SNe Ic-BL
} are associated with an LGRB. 
scenario invokes an asymmetric explosion that produces ejecta with an energy of $2 \times 10^{51} \mathrm{erg}$, total mass of $2 M_{\odot}$, and ${ }^{56} \mathrm{Ni}$ mass of $0.2 M_{\odot}$ (Höflich et al. 1999). However, all these models underestimate the luminosity at late times because the ejecta density is too low to sufficiently trap $\gamma$-rays from ${ }^{56} \mathrm{Co}$ decay (Sollerman et al. 2000; Patat et al. 2001). A similar discrepancy was found for SN 1997ef, which led Mazzali et al. (2000) to propose that the explosion may have been asymmetric.

Maeda et al. (2003) emphasise the inherent problem of spherically symmetric models to explain simultaneously the early-time and late-time light curve of SN 1998bw and other LGRB/SNe. They propose a two-component model with ${ }^{56} \mathrm{Ni}$ both located in the outer fast-moving ejecta and in the inner denser and slower ejecta. With this additional freedom, the model can explain the huge brightness at early times and sustained brightness at late times. A strong ejecta asphericity is also inferred by Maeda et al. $(2006,2008)$ from nebular phase spectra of SN 1998bw and other LGRB/SNe.

Fast rotation is thought to be central for the production of LGRB/SNe, both in the context of the collapsar model (Woosley 1993) and the proto-magnetar model (Wheeler et al. 2000; Bucciantini et al. 2008; Metzger et al. 2011). To preserve its original angular momentum, an initially fast-rotating single star must lose little mass. Furthermore, because of its redistribution within the star, angular momentum may be retained if the star does not evolve through a giant phase, which occurs if it evolves chemically homogeneously; this holds even in the absence of mass loss (Yoon \& Langer 2005; Woosley \& Heger 2006; Yoon et al. 2006). With the current understanding of hot star winds, this situation requires an evolution at low metallicity. An important corollary is that then the final mass is close to the initial mass. As discussed above, this poses serious concerns about the whole theory because the inferred ejecta mass of LGRB/SNe may be only a few $M_{\odot}$.

With the goal of reaching a final mass at collapse of about $10 M_{\odot}$ and keeping a lot of angular momentum, Woosley \& Heger (2006) evolved $12-16 M_{\odot}$ stars at very low metallicity. These stars die with lots of angular momentum but their final mass is low and their propensity for black hole $(\mathrm{BH})$ formation is questionable. Indeed, Dessart et al. (2012) argue that nearly all of these models are unlikely to form a $\mathrm{BH}$, primarily because their core structure (or compactness) is analogous to that of standard $15 M_{\odot}$ red supergiant (RSG) stars that are expected to produce SNe II-P. In the study of Woosley \& Heger (2006), only the more massive models (models 35OB-35OC) with a progenitor mass of $35 M_{\odot}$ have a huge compactness and may produce a $\mathrm{BH}$ if they do not explode through a magneto-rotational explosion first (Dessart et al. 2008). However, models 35OB-35OC die with a final mass of 20-30 $M_{\odot}$, which is incompatible with the inferences of Woosley et al. (1999) or Iwamoto et al. (1998) for SN 1998bw. The model of Woosley et al. (1999) leaves behind a neutron star or probably a magnetar, but not a BH. Yoon et al. (2006) studied the properties of low-metallicity fast-rotating massive stars. These stellar models evolve chemically homogeneously and retain a large amount of angular momentum, but they produce WR stars at death with a mass of $10-30 M_{\odot}$.

The question of the final WR mass is especially important. It is a fundamental characteristic of the progenitor, controls the ejecta properties, and is a likely determinant for the nature of the compact remnant. If one could invoke a large final WR mass for LGRB/SN progenitors, numerous concerns would immediately disappear for the production of a collapsar (Woosley 1993; MacFadyen \& Woosley 1999). First, such massive-star models are more likely to form BHs. As these massive stars no longer need to lose much mass they may retain a lot of angular momentum, although a low metallicity would still be needed to limit the wind mass-loss rate and to ensure a chemicallyhomogeneous evolution (to prevent a giant phase). Massive WRs are naturally encountered in the high-luminosity compact regions, which are the site of LGRB/SNe (see e.g. Le Floc'h et al. 2003; Fruchter et al. 2006; Modjaz et al. 2008).

Because of the complexity of LGRB/SNe, it is important to perform independent studies of their light curve and spectra with different tools and techniques. Even today, the bulk of the analyses of LGRB/SNe and standard SNe Ic are based on a simplistic 1D modelling of the light curve and/or no modelling of the spectra (for recent studies see e.g. Drout et al. 2011; D'Elia et al. 2015; Toy et al. 2016; Prentice et al. 2016; Volnova et al. 2017). There is a persisting controversy with the origin of LGRB/SNe. Some recent studies argue for the protomagnetar model (Mazzali et al. 2014; Wang et al. 2017) and it is therefore necessary to assess independently the LGRB/SN ejecta properties and address the pros and cons of the collapsar and proto-magnetar models.

Here, we present 1D time-dependent radiative transfer models based on the explosion of a set of carbon-rich WR stars, which result from the evolution of a rotating or non-rotating $40 M_{\odot}$ star on the zero-age main sequence (ZAMS). Unlike previous studies, our approach computes the evolution of the full ejecta from early to late times and generates the frequencydependent emergent flux, from which both multi-band light curves and UV/optical/near-IR spectra can be simultaneously extracted; the same physics is used to compute both photometric and spectroscopic observables (Hillier \& Dessart 2012). This is the first time such simulations are presented.

The paper is organised as follows. In the next section, we present the numerical approach, including the simulation of the pre-SN evolution, explosion dynamics, and radiative transfer. These simulations are similar to the CO core explosion models of Woosley et al. (1999), with the exception of our BH-forming SN model, but the observables are computed with greater physical consistency. In Sect. 3, we discuss the key photometric and spectroscopic properties of these models and confront them to the observations of SN 1998bw. We confirm that the early-time light curve requires a large ejecta energy-to-mass ratio and a large ${ }^{56} \mathrm{Ni}$ mass and that such a model fails to match the latetime light curve. Further, our models that match the early-time spectra fail to match even approximately the late-time spectra, and vice-versa. In support of previous studies, we find numerous lines of evidence that the SN 1998bw may be axisymmetric, both from the spectra and the light curve and both from early times and late times. In Sect. 4, we present our conclusions. This study is a stepping stone for forthcoming radiative transfer simulations in $2 \mathrm{D}$.

\section{Numerical setup}

The simulations we present in this study are all based on a $40 M_{\odot}$ progenitor star evolved from the ZAMS until the onset of core collapse with the code MESA (Paxton et al. 2011, 2013, 2015), version 5696. The metallicity is set to 0.0162 for the solar metallicity models and 0.002 for the low metallicity model. We consider models with and without rotation on the ZAMS. The effects of rotation on stellar structure, chemical mixing, angular momentum transport, and mass loss are accounted for according to the methods available in MESA (Paxton et al. 2015). All models correspond to single stars, so that any mass loss takes 
Table 1. Properties of the models evolved with MESA, some corresponding to the initial conditions on the ZAMS and others to the onset of core collapse.

\begin{tabular}{lcccccccccccccc}
\hline \hline Model & $Z_{\text {init }}$ & $\Omega / \Omega_{\text {crit }}$ & $\begin{array}{c}\text { Age } \\
{[\mathrm{Myr}]}\end{array}$ & $\begin{array}{c}T_{\text {eff }}[\mathrm{kK}] \\
{[\mathrm{kK}}\end{array}$ & $\begin{array}{c}L_{\star} \\
{\left[L_{\odot}\right]}\end{array}$ & $\begin{array}{c}R_{\star} \\
{\left[R_{\odot}\right]}\end{array}$ & $\begin{array}{c}M_{\text {final }} \\
{\left[M_{\odot}\right]}\end{array}$ & $\left.\begin{array}{c}M_{\mathrm{Si}, \mathrm{c}} \\
{\left[M_{\odot}\right]}\end{array}\right] M_{\mathrm{Fe}, \mathrm{c}}$ & $X_{\mathrm{He}, \mathrm{s}}$ & $X_{\mathrm{C}, \mathrm{s}}$ & $X_{\mathrm{N}, \mathrm{s}}$ & $X_{\mathrm{O}, \mathrm{s}}$ & $X_{\mathrm{Si}, \mathrm{s}}$ \\
\hline r0 & 0.0162 & 0 & 5.06 & 166 & $3.39(5)$ & 0.70 & 11.4 & 1.59 & 1.57 & $1.88(-1)$ & $5.14(-1)$ & 0.0 & $2.79(-1)$ & $5.03(-4)$ \\
r4 & 0.0162 & 0.4 & 5.44 & 200 & $3.28(5)$ & 0.48 & 10.0 & 2.01 & 1.82 & $1.75(-1)$ & $5.20(-1)$ & 0.0 & $2.86(-1)$ & $5.03(-4)$ \\
r6 & 0.0162 & 0.6 & 6.25 & 146 & $1.79(5)$ & 0.67 & 6.6 & 1.67 & 1.55 & $2.91(-1)$ & $5.38(-1)$ & 0.0 & $1.52(-1)$ & $5.02(-4)$ \\
r6z & 0.002 & 0.6 & 7.37 & 145 & $2.88(5)$ & 0.86 & 9.7 & 2.15 & 1.87 & $9.98(-1)$ & $8.57(-5)$ & $1.55(-3)$ & $2.41(-5)$ & $3.76(-5)$ \\
\hline
\end{tabular}

Notes. All models start from a $40 M_{\odot}$ star on the ZAMS, but with different initial rotation rates and metallicities.

the form of a radiation-driven wind. The wind mass loss rate is determined by the "Dutch" recipe in MESA, combining the values from Glebbeek et al. (2009), Nieuwenhuijzen \& de Jager (1990), Nugis \& Lamers (2000), and Vink et al. (2001), with a coefficient $\eta=1.4$. Convection is followed according to the Ledoux criterion with a mixing length parameter $\alpha_{\mathrm{MLT}}=3$, a semi-convection efficiency parameter $\alpha_{\mathrm{sc}}=0.1$ (Paxton et al. 2015, Eq. (12)), and an exponential overshoot with parameter $f=0.008$ (Paxton et al. 2011, Eq. (2)).

Since all models are derived from a $40 M_{\odot}$ star on the ZAMS, our nomenclature distinguishes models according to the initial rotation rate and the metallicity. We use $\mathrm{r} 0$ to refer to a model without rotation and $\mathrm{r} 4$ (r6) for a model spinning initially at $40 \%$ $(60 \%)$ of the critical angular velocity $\Omega_{\text {crit }}$ at the surface. We also include one fast-rotating model at a tenth of the solar metallicity (model r6z). By the time of core collapse, all models are $\mathrm{H}$ free, but they retain a small amount of $\mathrm{He}$ within the $\mathrm{CO}$ core. These models would correspond to C-rich (or O-rich) WR stars (Maeder \& Meynet 1994; Langer et al. 1994; Groh et al. 2013). Some model properties at the onset of core collapse are given in Table 1.

Using V1D (Livne 1993; Dessart et al. 2010b,a), the explosion is triggered by means of a piston to produce an ejecta kinetic energy of about 4 and $12 \times 10^{51} \mathrm{erg}$; models are identified by suffixes e 2 and e 4 in order of explosion energy. The code treats nuclear burning but the explosive nucleosynthesis yields a ${ }^{56} \mathrm{Ni}$ mass that depends sensitively on the piston location (in mass space) and on the piston trajectory and velocity. Consequently, our simulations produce a sizeable scatter in ${ }^{56} \mathrm{Ni}$ mass, which is not necessarily strongly correlated with explosion energy, with values in the range $0.085-0.696 M_{\odot}$. In all models except r6e4BH, the piston mass cut is located at the edge of the Fe core, somewhere between 1.5-2.0 $M_{\odot}$ (producing a neutron star remnant). In model r6e $4 \mathrm{BH}$, we place it at $4 M_{\odot}$ to produce a $\mathrm{BH}$ and explore the effect of a much lower ejecta mass (i.e. $2.55 M_{\odot}$, compared to $5-10 M_{\odot}$ for the rest of the sample) on the $\mathrm{SN}$ radiation. This could occur in a progenitor star whose innermost stable circular orbit is located at $4 M_{\odot}$. The sensitivity of our results to $\mathrm{BH}$ mass and for a set of fast-rotating carbon-rich WR progenitors will be presented in a forthcoming paper.

In models forming a neutron star, the piston is given a fixed outwards velocity of $15000-30000 \mathrm{~km} \mathrm{~s}^{-1}$ until the total energy of the stellar envelope equals the desired asymptotic kinetic energy; the piston is set to rest thereafter. In model r6e4BH, we instead adopt the same piston trajectory as in KEPLER (Woosley \& Weaver 1995). Placing the piston at $M_{\text {piston }}=4 M_{\odot}$, we first drive the piston inwards for $30 \mathrm{~s}$ to simulate the envelope collapse into the gravitational potential well. When the piston reaches down to $R_{\min }=500 \mathrm{~km}$, we drive the piston outwards at an initial speed $V_{0}$ of $40000 \mathrm{~km} \mathrm{~s}^{-1}$. The piston trajectory follows $\mathrm{d} R_{\text {piston }} / \mathrm{d} t=\left[\alpha G M_{\text {piston }}\left(1 / R_{\text {piston }}-1 / R_{\min }\right)+V_{0}^{2}\right]^{1 / 2}$, where $\alpha=0.4 V_{0}^{2} / M_{\text {piston }}$ (see Woosley \& Weaver 1995, for discussion). The collapse phase brings the inner envelope to small radii, compressing the material to large densities. When the piston moves outwards, the large post-shock temperatures necessary for the synthesis of a large mass of ${ }^{56} \mathrm{Ni}$ are easily produced.

The full set of models includes r0e2, r0e4, r4e4, r6e4, r6ze4, and r6e $4 \mathrm{BH}$. Once the explosive nucleosynthesis is over, we mix ${ }^{56} \mathrm{Ni}$, and only ${ }^{56} \mathrm{Ni}$; we do not mix other species; we do, however, adjust the $\mathrm{O}$ mass fraction to keep the sum of mass fractions equal to unity ${ }^{2}$. Mixing ${ }^{56} \mathrm{Ni}$ captures the influence of decay heating on the light curve. By not altering other species, we avoid artificially introducing a microscopic mixing that probably does not occur in real SN explosions (see e.g. Jerkstrand et al. 2011; Wongwathanarat et al. 2015). When mixing is applied to all species, we find little difference in the SN radiation, probably because these progenitors are quasi-homogeneous $\mathrm{CO}$ cores; this is in stark contrast with simulations of SNe II-P (Lisakov et al. 2017).

The explosion models computed with V1D are then remapped into CMFGEN (Hillier \& Dessart 2012) at 1-4d after explosion. The numerical procedure is the same as the one used recently in Dessart et al. $(2015,2016)$. Because of the large abundance of intermediate mass elements (IMEs) and Fe-group elements (IGEs) in these ejecta, we use the same model atoms for metals as used in Dessart et al. (2014b); it is particularly important to test for the presence of Co III lines (Dessart et al. 2014a). The Co III $5888 \AA$ line may be seen and could be an important diagnostic because SNe Ic-BL are believed to synthesise a mass of ${ }^{56} \mathrm{Ni}$ comparable to that of standard $\mathrm{SNe}$ Ia (Scalzo et al. 2014). We show the ejecta composition (for a selection of important species) for models $\mathrm{r} 0 \mathrm{e} 2$ and $\mathrm{r} 6 \mathrm{e} 4 \mathrm{BH}$ in Fig. 1. Additional ejecta properties are summarised in Table 2.

Our set of simulations was not carried out to match a specific SN Ic, SN Ic-BL, or LGRB/SN. Rather, we cover a range of progenitor and ejecta properties and study their impact on observables. We then confront these results to the photometric and spectroscopic evolution of SN 1998bw with data of Galama et al. (1998), McKenzie \& Schaefer (1999), and Patat et al. (2001). We use an extinction $A_{V}=0.2 \mathrm{mag}$, redshift of 0.00867 , and distance modulus of 32.89 mag (Patat et al. 2001). We set the explosion time to the time of the LGRB detection (MJD 50 929.4; Galama et al. 1998).

\footnotetext{
2 In practice, starting at the base of the ejecta, we reset the ${ }^{56} \mathrm{Ni}$ mass fraction $X_{i}$ in each shell $i$ of mass $\delta m_{i}$ to $\sum_{k=i}^{j} X_{k} \delta m_{k} / \Delta m$, where $\Delta m=$ $\sum_{k=i}^{j} \delta m_{k}$. In all models, we use $\Delta m=0.6 M_{\odot}$, except for models r6e4 and r6e $4 \mathrm{BH}$ for which we use $0.3 M_{\odot}$. If the sum of mass fractions in mass shell $i$ is greater than unity, we scale all mass fractions so that the sum is unity. If it is less than unity, we increase the local $\mathrm{O}$ mass fraction so that the sum is unity. In practice, this adjustment is minor.
} 
Table 2. Summary of ejecta properties used as initial conditions for the CMFGEN calculations.

\begin{tabular}{lcccccccccc}
\hline \hline Model & $\begin{array}{c}M_{\mathrm{r}} \\
{\left[M_{\odot}\right]}\end{array}$ & $\begin{array}{c}M_{\mathrm{e}} \\
{\left[M_{\odot}\right]}\end{array}$ & $\begin{array}{c}E_{\mathrm{kin}} \\
{\left[10^{51} \mathrm{erg}\right]}\end{array}$ & $\begin{array}{c}\left\langle V_{\mathrm{m}}\right\rangle \\
{\left[\mathrm{km} \mathrm{s}^{-1}\right]}\end{array}$ & $\begin{array}{c}\mathrm{He} \\
{\left[M_{\odot}\right]}\end{array}$ & $\begin{array}{c}\mathrm{C} \\
{\left[M_{\odot}\right]}\end{array}$ & $\begin{array}{c}\mathrm{O} \\
{\left[M_{\odot}\right]}\end{array}$ & $\begin{array}{c}\mathrm{Si} \\
{\left[M_{\odot}\right]}\end{array}$ & $\begin{array}{c}\mathrm{Ca} \\
{\left[M_{\odot}\right]}\end{array}$ & $\begin{array}{c}{ }^{56} \mathrm{Ni} \\
{\left[M_{\odot}\right]}\end{array}$ \\
\hline r0e2 & 1.71 & 9.69 & 4.12 & 6530 & 0.181 & 1.326 & 5.471 & 0.112 & 0.0061 & 0.122 \\
r0e4 & 1.54 & 9.86 & 12.31 & 11210 & 0.205 & 1.298 & 5.590 & 0.181 & 0.0098 & 0.172 \\
\hline r4e4 & 1.88 & 8.12 & 13.44 & 12900 & 0.286 & 1.302 & 3.852 & 0.458 & 0.0410 & 0.583 \\
\hline r6e4 & 1.62 & 4.97 & 12.41 & 15840 & 0.324 & 1.051 & 2.072 & 0.315 & 0.022 & 0.300 \\
\hline r6ze4 & 1.99 & 7.70 & 13.70 & 13370 & 1.453 & 0.822 & 3.017 & 0.429 & 0.0386 & 0.696 \\
\hline r6e4BH & 4.04 & 2.55 & 11.63 & 21420 & 0.456 & 0.910 & 0.515 & 0.051 & 0.0036 & 0.435 \\
\hline
\end{tabular}

Notes. All models derive from pre-SN MESA models named r0, r4, r6, or r6z (see Table 1). Model r6e4BH forms a $4.04 M_{\odot} \mathrm{BH}-$ all other models leave behind a 1.6-1.9 $M_{\odot}$ neutron star.
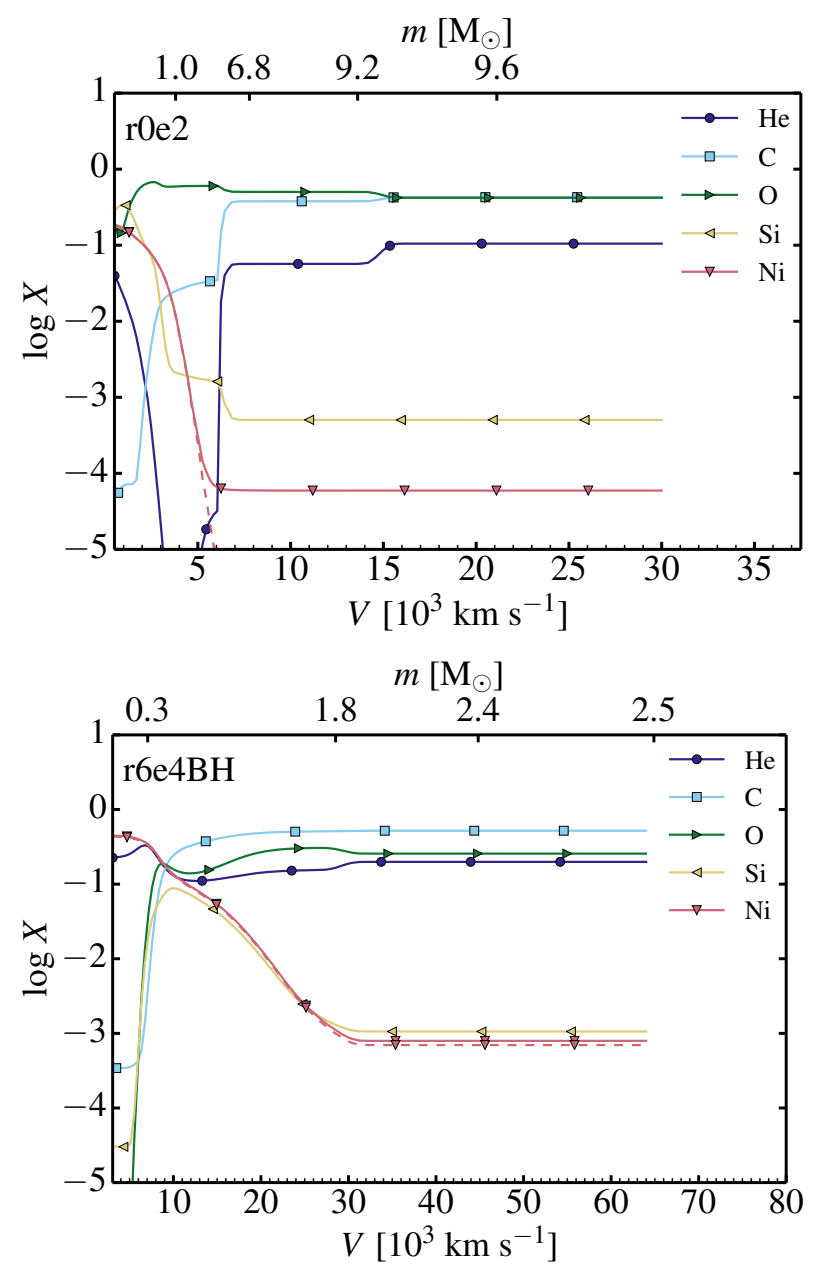

Fig. 1. Ejecta composition at the start of the CMFGEN simulations for models r0e2 (top; $M_{\mathrm{e}}=9.69 M_{\odot}$; post-explosion time of $\sim 1.1 \mathrm{~d}$ ) and r6e4BH (bottom; $M_{\mathrm{e}}=2.55 M_{\odot}$; post-explosion time of $\sim 4.6 \mathrm{~d}$ ). The dashed line corresponds to the ${ }^{56} \mathrm{Ni}$ mass fraction at the given time. The top axis gives the Lagrangian mass coordinate in the ejecta (zero corresponds to the base of the ejecta). In model r6e $4 \mathrm{BH}$, most of the O-rich shell has been accreted into the $4 M_{\odot} \mathrm{BH}$. In particular, the outer layers where $\mathrm{He}$ is present now overlap with the ${ }^{56} \mathrm{Ni}$-rich regions.

\section{Results}

\subsection{Photometry}

Using the UBVRI photometry for both the observations of SN 1998bw and for our set of models, we convert these

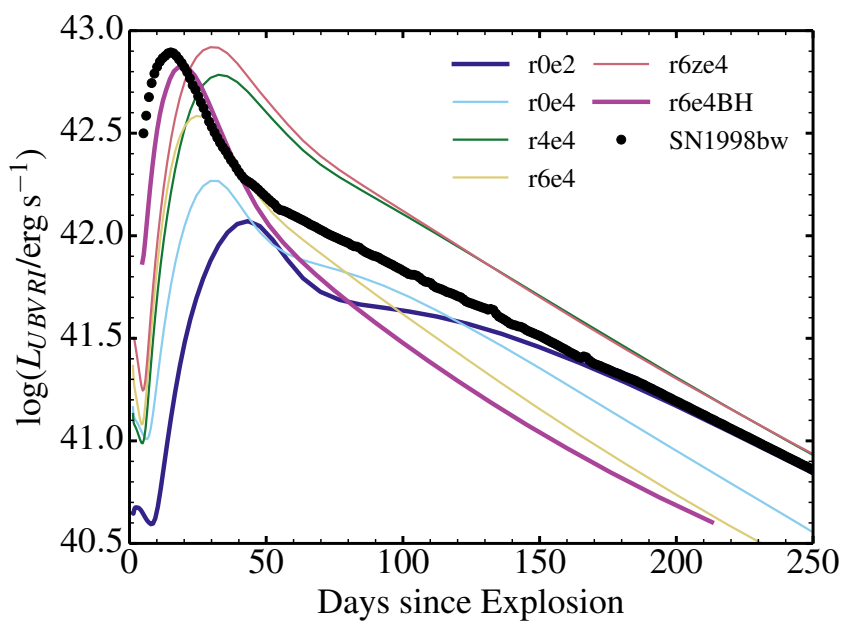

Fig. 2. Evolution of $L_{U B V R I}$ for SN 1998bw and for the set of single WR star explosion models (with and without rotation; see Tables 1 and 2 for details). Models r4e4, r6e4, r6ze4, and r6e $4 \mathrm{BH}$ come close to match the luminosity around maximum but overestimate the rise time and/or underestimate the luminosity at late times. Less energetic and more massive models with a lower ${ }^{56} \mathrm{Ni}$ mass (models r0e2 and r0e4) are in better agreement at late times, but they fail to match the phase around maximum (see Sect. 3.1 for discussion and details on how we calculate $\left.L_{U B V R I}\right)$.

magnitudes to fluxes and integrate to yield a luminosity $L_{U B V R I}{ }^{3}$. Repeating the process from early to late times, we build optical light curves (Fig. 2).

Our set of six models may be split in two separate groups. The first group contains models $\mathrm{r} 0 \mathrm{e} 2$ and $\mathrm{r} 0 \mathrm{e} 4$, which correspond to a massive ejecta with modest amounts of ${ }^{56} \mathrm{Ni}(0.12$ and $\left.0.17 M_{\odot}\right)$, and kinetic energies of 4.1 and $12.3 \times 10^{51} \mathrm{erg}$. Both models are too faint to match the peak luminosity of SN 1998bw. These models also have a very long rise time to maximum from $\sim 43 \mathrm{~d}$ (r0e2) down to $\sim 31 \mathrm{~d}$ (r0e4). Such massive ejecta, which are typical of what galactic WR stars may produce (see Crowther 2007, for a review), yield SN radiation properties that are incompatible with the inferred rise times and light curve widths of SNe Ic (see e.g. Drout et al. 2011), unless they have an explosion energy well in excess of $10^{52} \mathrm{erg}$. The exceptional conditions required to produce such extreme energies are unlikely to be realised with the frequency at which standard SNe Ic occur. Super-solar metallicity may be needed to produce the low to moderate mass WR progenitors required to match the observed SN Ic properties (Georgy et al. 2009; Yoon 2015).

3 In practice, we use the same procedure as in Blondin et al. (2013); for additional details, see e.g. Valenti et al. (2008). 

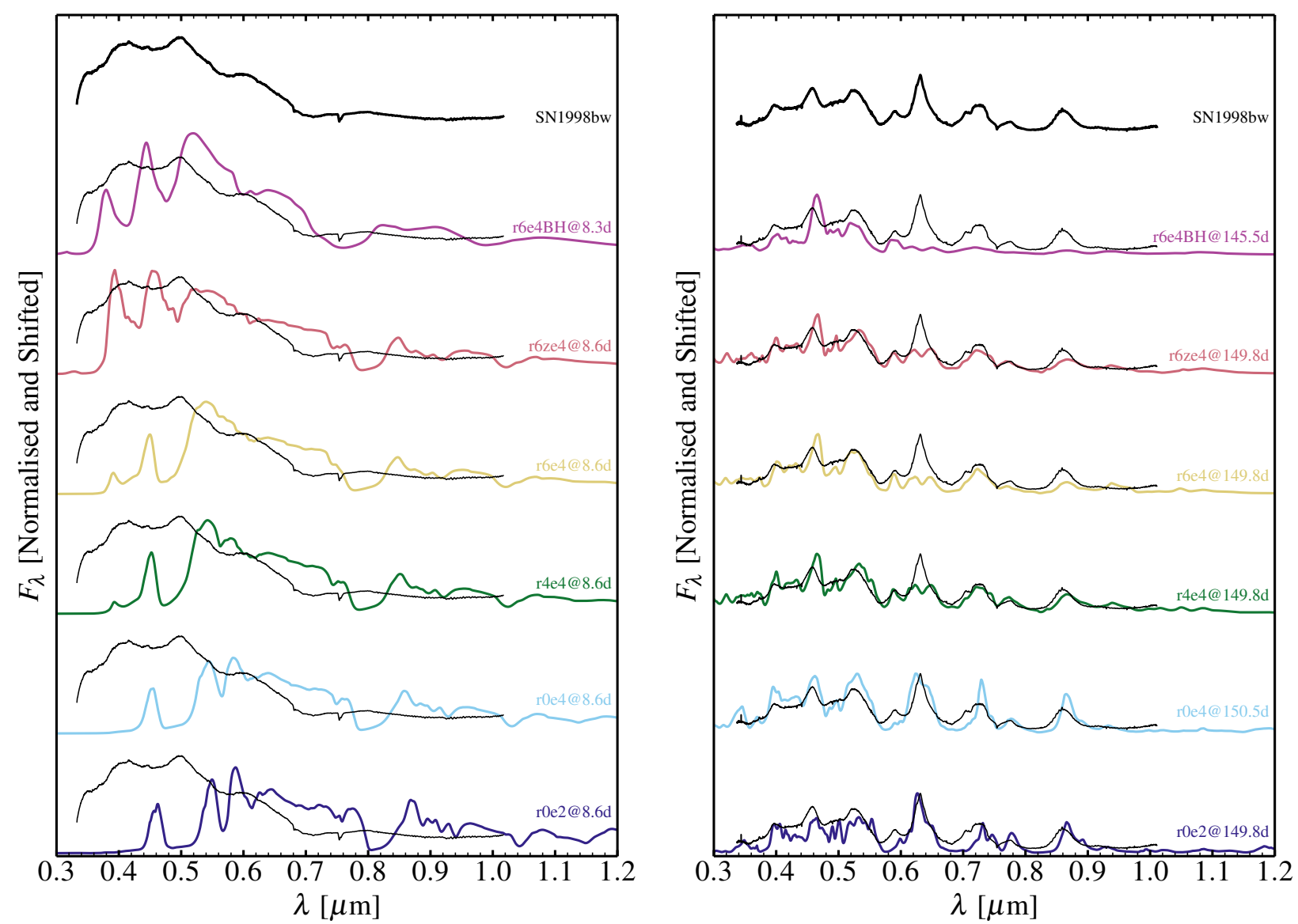

Fig. 3. Optical spectra at early times (left) and late times (right) for SN 1998bw corrected for extinction and redshift; the epochs are 8.4 and $139.4 \mathrm{~d}$ after the LGRB detection. In addition, optical spectra at early times and late times are shown for a selection of our models. The post-explosion epoch is indicated next to each spectrum. The (normalized and shifted) observed spectrum is shown at the top as well as overplotted as a thin black line over each model; the normalization is at $6300 \AA$ for the left panel, and at the location of maximum flux for the right panel. Models with a larger (smaller) $E_{\mathrm{kin}} / M_{\mathrm{e}}$ come closer to matching the spectrum of SN $1998 \mathrm{bw}$ at early (late) times. The BH forming SN model r6e4BH matches best the spectral properties of SN 1998bw at early times, but models r0e2/r0e4 match better the nebular-phase spectrum. No model matches both the early- and late-time spectra of SN 1998bw.

The second set contains models with lower ejecta masses $\left(\sim 3-8 M_{\odot}\right)$, large explosion energies $\left(\sim 12 \times 10^{51} \mathrm{erg}\right)$, and large ${ }^{56} \mathrm{Ni}$ masses $\left(0.3-0.7 M_{\odot}\right)$. The higher the ${ }^{56} \mathrm{Ni}$ mass, the greater the bolometric luminosity at maximum (compare model r6ze4 to model r6e4). The larger the $M_{\mathrm{e}}$, the longer the rise time to maximum and the broader the light curve (cf. model r6ze4 to r6e $4 \mathrm{BH})$. The greater the $E_{\mathrm{kin}} / M_{\mathrm{e}}$, the faster is the decline rate at late times. These trends are the same as those obtained in a previous grid of models of $\mathrm{SNe} \mathrm{IIb} / \mathrm{Ib} / \mathrm{Ic}$ (Dessart et al. 2015, 2016).

The model that best matches the light curve of SN 1998bw at early times is the BH forming model. The model peaks at $20 \mathrm{~d}$, only $5 \mathrm{~d}$ later than observed, but it is also a little too faint. The model light curve width is comparable to that of SN 1998bw. However, the low ejecta mass, which allows the very fast rise to maximum, causes an enhanced $\gamma$-ray escape at late times, producing a low SN luminosity. Models r4e4 and r6ze4 are less discrepant at late times but they overestimate the rise time to maximum and the light curve width. Model r6e4 is too faint at most times; it contains too little ${ }^{56} \mathrm{Ni}$ initially.

In contrast, model $\mathrm{r} 0 \mathrm{e} 2$ fits the epochs poorly around maximum but it follows closely the optical luminosity of SN 1998bw at nebular times, despite its modest ejecta energetics and ${ }^{56} \mathrm{Ni}$ mass of only $0.122 M_{\odot}$. The lower decay power yields a much higher luminosity than in model r6e $4 \mathrm{BH}$ because of the near complete trapping of $\gamma$-rays.
Hence, we corroborate the results of Woosley et al. (1999). To come close to matching the early-time light curve, a spherically symmetric model needs a huge ejecta kinetic energy well in excess of $10^{52} \mathrm{erg}, 0.5 M_{\odot}$ of ${ }^{56} \mathrm{Ni}$, and a low- to moderate-ejecta mass. But, as emphasised by Maeda et al. (2003), these highly energetic ejecta tend to yield a luminosity at late times that is too faint or decreases over time with the wrong rate.

\subsection{Spectroscopy}

We now discuss the spectral evolution of our set of models from pre-maximum to the nebular phase and compare this evolution to the observations of SN 1998bw. Contrary to all previous studies that focused on snapshots (see e.g. Nakamura et al. 2001), this work presents the first spectral model sequences based on non-LTE, time-dependent radiative transfer simulations for SNe Ic-BL.

\subsubsection{Early times}

Figure 3 compares the optical spectra for our model set at $\sim 8 \mathrm{~d}$ and $\sim 135 \mathrm{~d}$ after explosion with the contemporaneous observations of SN 1998bw. At early times, all models fail to reproduce the width of the observed absorption features, despite their large 
$E_{\text {kin }} / M_{\mathrm{e}}$ ratio, even for model r6e4BH. The model spectra have both narrower line features and stronger absorption troughs than SN 1998bw. Hence, this suggests an even larger energy per unit mass is needed to match the observations.

Our models are also too red, except for the low-metallicity model r6ze4, which comes close to matching the optical colours of SN 1998bw at $8 \mathrm{~d}$. There is a complicated combination of effects since model r6ze4 has a low primordial metallicity but this model is also very metal rich owing to the secular and explosive nucleosynthesis that took place. At $8 \mathrm{~d}, \mathrm{Ni} / \mathrm{Co}$ do not greatly pollute the outer ejecta and they have not yet decayed to raise the Fe abundance sizeably. Hence, the Fe mass fraction is down by a factor of ten compared to solar in the outer ejecta. At early times, a lower primordial metallicity can therefore produce a bluer SN.

As illustrated for models $\mathrm{r} 0 \mathrm{e} 2$ and $\mathrm{r} 6 \mathrm{e} 4 \mathrm{BH}$ in the appendix (Figs. A.1-B.2), the early-time optical spectra show neutral or once ionised lines of IMEs. The larger the ratio $M\left({ }^{56} \mathrm{Ni}\right) / M_{\mathrm{e}}$, the larger the ionisation in the spectrum formation region. In model $\mathrm{r} 0 \mathrm{e} 2$, the spectra show the presence of lines from C I (red part of the optical), O I, Na I D, Mg I Ca II, together with strong blanketing from Fe I shortward of $5500 \AA$. There is no sizeable contribution from Si II $6355 \AA$. In model r6e4BH, the ionisation is much higher and the expansion rate in the spectrum formation is larger. We therefore see broad lines of C I, O I, Si II, Ca II, and Fe II.

While all the features seen in model r6e4BH are compatible with those observed in the spectrum of SN 1998bw at $8.4 \mathrm{~d}$, they are too narrow. Even if we were to scale this model to the $10 M_{\odot}$ ejecta required by Iwamoto et al. (1998), with an explosion energy of $4.6 \times 10^{52} \mathrm{erg}$, the lines would still be too narrow. Furthermore, this energy approaches the theoretical upper limit of $\sim 10^{53} \mathrm{erg}$, which is the gravitational binding energy of a typical neutron star.

Nakamura et al. (2001) modelled the early time spectra of SN 1998bw and found similar properties for the spectrum formation at early times, including very fast expansion (with absorption extending out to $60000 \mathrm{~km} \mathrm{~s}^{-1}$ in some lines) and a composition dominated by IMEs. Their line identifications are identical to ours around the epoch of maximum. They note that the line absorptions are better fitted when invoking a flatter density distribution at large velocity (they argue for a power law with a density exponent of -6 , while we have a value of -7.5 in model r6e4BH; Fig. 4).

None of our models shows the strong optical He I lines characteristic of $\mathrm{SNe} \mathrm{Ib}$ - all our models produce $\mathrm{SNe}$ Ic (or $\mathrm{SNe}$ Ic-BL), although they all contain some He. The total He mass is in the range $0.18-1.4 M_{\odot}$, with a surface He mass fraction of $20 \%$ (model r0, r4, r6) and 98\% (model r6z). However, model r6e4BH shows the presence of He I $10830 \AA$, although the line is weak at this early time. Hence, He deficiency is not mandatory for producing a SN Ic or a SN Ic-BL (the same result is obtained for the SN Ic ejecta models $5 \mathrm{p} 11 \mathrm{Ax} 1 / 5 \mathrm{p} 11 \mathrm{Ax} 2$ in Dessart et al. 2015).

\subsubsection{Late times}

In contrast to early times, our model set produces a larger diversity in spectral appearance at late times (Fig. 3, right panel). This results from the differences in ionisation (greater in ejecta with a larger $M\left({ }^{56} \mathrm{Ni}\right) / M_{\mathrm{e}}$ ratio) and composition.

Models with a low $M\left({ }^{56} \mathrm{Ni}\right) / M_{\mathrm{e}}$ ratio (r0e2 and $\left.\mathrm{r} 0 \mathrm{e} 4\right)$ show a spectrum dominated by O I lines at 6300-6363 $\AA$ (forbidden doublet) and $7774 \AA$, Na I D, the Ca II $7300 \AA$ doublet and the Ca II near-IR triplet around $8500 \AA$, and lines of Fe II. In the

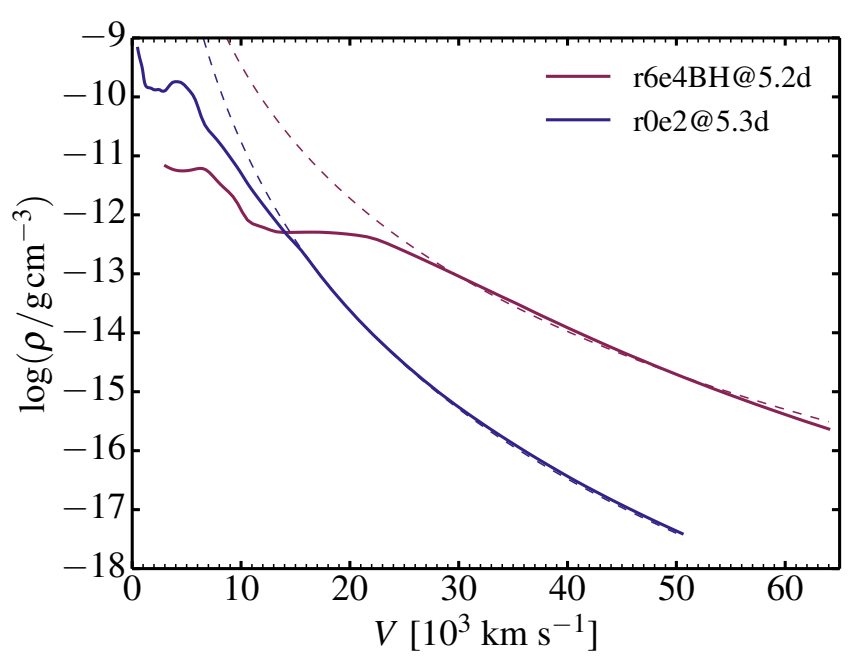

Fig. 4. Comparison of the density vs. velocity for models r6e4BH and $\mathrm{r} 0 \mathrm{e} 2$ at $\sim 5 \mathrm{~d}$ after explosion. The dashed lines represent fits to the density distribution using the expression $\log \rho(V)=\log \rho(V=$ $\left.V_{0}\right)+N_{\rho} \log \left(V_{0} / V\right)$, where $V_{0}=30000 \mathrm{~km} \mathrm{~s}^{-1}$ and $N_{\rho}=7.5$ for model r6e4BH and $V_{0}=20000 \mathrm{~km} \mathrm{~s}^{-1}$ and $N_{\rho}=9.5$ for model r0e2. The greater the energy of the explosion, the more material is present at large velocity. In spherical symmetry, radiation hydrodynamics cannot produce high density regions at both low and high velocity. However, by adopting an axisymmetric configuration it is possible, for example to have dense material at high velocities in the polar direction, and at low velocity in the equatorial regions.

blue part of the optical, we see the overlapping contributions of forests of lines from Ti II, Fe II, as well as strong absorptions, for example from $\mathrm{Ca}$ II $\mathrm{H} \& \mathrm{~K}$.

In contrast, model r6e4BH shows a dominance of IGEs, with the strong Fe II, Fe III, and Co III lines (Fig. 3), and a spectral energy distribution that peaks in the blue part of the optical. There is no emission associated with O I 6300-6364 $\AA$, but we see a sizeable emission in [Co III] $5888 \AA$, as also observed in SNe Ia (Dessart et al. 2014a). Overall, this model spectrum resembles a Type Ia spectrum at late times, which is not surprising given the model properties $\left(M_{\mathrm{e}}=2.55 M_{\odot}, M\left({ }^{56} \mathrm{Ni}\right)=0.435 M_{\odot}\right)$.

Model r6e4BH, despite its suitability for the early-time properties of SN 1998bw, is strongly discrepant at late times. In contrast, model r0e2 matches closely the spectral properties of SN 1998bw at nebular times, even though it corresponds to the explosion of a massive progenitor yielding a low-to-moderate energy and a low ${ }^{56} \mathrm{Ni}$ mass $\left(M_{\mathrm{e}}=9.69 M_{\odot}, E_{\text {kin }}=4.12 \times\right.$ $\left.10^{51} \mathrm{erg}, M\left({ }^{56} \mathrm{Ni}\right)=0.122 M_{\odot}\right)$.

From the above discussion we see that while our models reproduce some of the features of the optical light curve and spectra of SN 1998bw at specific epochs, all models also show significant discrepancies when compared to observations at all epochs.

\section{Discussion and conclusions}

We have presented physically consistent explosion models for rotating and non-rotating C/O-rich WR stars. Starting at $1-4 \mathrm{~d}$ after explosion, we follow each model with the 1D timedependent radiative transfer code CMFGEN, allowing for thermal heating and non-thermal processes associated with the radioactive decay of ${ }^{56} \mathrm{Ni}$ and ${ }^{56} \mathrm{Co}$. Our approach allows the simultaneous computation of bolometric, multi-band light curves and spectra for models of SNe Ic-BL. While our modelling confirms some results from previous work, it also conflicts with numerous recent studies of LGRB/SNe. 
We find that a high energy explosion yielding a high $E_{\mathrm{kin}} / M_{\mathrm{e}}$ can approximately reproduce the light curve properties around maximum (rise time, peak brightness, and light curve width) as well as the very broad optical lines (associated with Fe II, Si II, or Ca II) observed in SN 1998bw. But this model fails at late times because it underestimates the luminosity and yields spectra dominated by metal lines without the presence of for example O I 6300-6364 A. Conversely, we find that a lower energy explosion yielding a lower $E_{\text {kin }} / M_{\mathrm{e}}$, which fails at early times, produces a satisfactory match to both the observed luminosity and optical spectra at nebular times.

Maeda et al. (2003) suggested that the observed light curve of SN 1998bw can be reconciled by invoking a highly energetic ejecta with a distribution of ${ }^{56} \mathrm{Ni}$ in two concentric shells at low and high velocity and a high density inner ejecta. In our work, and as illustrated in Fig. 4, we cannot reproduce this configuration because a high energy explosion model (e.g. r6e4BH) has little or no material at low velocity, while a low energy explosion has little or no material at high velocity (e.g. r0e2); the ejecta structure with two concentric and spherical shells proposed by Maeda et al. (2003) does not appear to be hydrodynamically feasible. In other words, a spherically symmetric explosion model cannot have simultaneously a large density at low and high velocity. A simple way out of this conundrum is to argue that the explosion at the origin of SN 1998bw is highly asymmetric, yielding, for example, a prolate (oblate) density distribution along the pole (equator). Such a morphology has been proposed by Maeda et al. (2006, 2008) based on modelling of nebular phase spectra. We propose here that evidence for asymmetry is visible at all times, both in the photometry and in the spectra.

Instead of a model with two concentric shells of ${ }^{56} \mathrm{Ni}$ located at low and high velocities, the ejecta may be axisymmetric with fast material (high energy per unit mass with high relative abundance of ${ }^{56} \mathrm{Ni}$ with respect to other species) limited to a small/moderate solid angle around our line of sight (and possibly 180 deg away from our line of sight), and slower material (lower energy per unit mass with a lower relative abundance of ${ }^{56} \mathrm{Ni}$ with respect to other species) lying along lower latitudes. This hybrid scenario is qualitatively similar to the axisymmetric explosions of MacFadyen \& Woosley (1999) or Maeda et al. (2002).

For the fast material along the pole, the ejecta properties could correspond to model r6e4BH, but limited to a small solid angle. At early times, the ejecta is optically thick and we are mostly sensitive to material along our line of sight. Consequently, the total mass of the very fast material and of its ${ }^{56} \mathrm{Ni}$ content are reduced to perhaps a tenth of the respective masses in the spherical ejecta model $\mathrm{r} 6 \mathrm{e} 4 \mathrm{BH}$, if this region occupies a tenth of the volume of this spherical model. The radiation from this material is not beamed by a relativistic effect, but it is non-isotropic and biased in the radial direction along which the photon mean free path is the greatest. Thus at early times, the spectra would form primarily in the optically thick column of fast-moving material along our line of sight, resembling the properties of our (1D) model r6e4BH. At late times, the ${ }^{56} \mathrm{Ni}$ at large velocity contributes negligibly to the light curve (because of $\gamma$-ray escape) and also because the corresponding mass is lower than the $0.5 M_{\odot}$ value of the corresponding spherical model; how much lower depends on the opening angle for the fast material and may vary from case to case.

This model reproduces roughly the salient features seen in the early-time spectra of SN 1998bw, with very broad Fe II and Si II lines, a broad feature in the red from the blended absorption of O I-Ca II. The key here is that the huge energy and huge ${ }^{56} \mathrm{Ni}$ mass inferred to match SN 1998bw arise when spherical symmetry is assumed. The early-time light curve and spectra may instead be reproduced by an asymmetric ejecta, with a much lower energy and ${ }^{56} \mathrm{Ni}$ mass for the fast moving material because it is limited to a small solid angle.

The bulk of the mass located at lower velocities could correspond to an ejecta mass of $10 M_{\odot}$ with an energy of a few $10^{51} \mathrm{erg}$ and $0.1 M_{\odot}$ of ${ }^{56} \mathrm{Ni}$. Our model rOe2 might be a rough representation of this inner ejecta in SN $1998 \mathrm{bw}$. Only $0.1 M_{\odot}$ of ${ }^{56} \mathrm{Ni}$ may be needed to reproduce the luminosity of SN 1998bw at late times (Fig. 2) because the denser, slower, and more massive ejecta leads to the more efficient trapping of $\gamma$-rays. The large $\mathrm{O}$ mass and low ionisation conditions lead to the production of a strong O I 6300-6364 $\AA$ line, as observed. In contrast, model r6e4BH shows only lines of IGEs at late times and no O I line emission. With both models combined, we can explain the simultaneous presence of Fe III, Co III, and O I lines at nebular times. Figure 5 illustrates how an asymmetric model may reproduce the spectral observations of SN 1998bw, resembling model r6e4BH at $\$ 70 \mathrm{~d}$ and model r0e2 later on.

Allowing for asymmetry, it is possible for LGRB/SN progenitors to be massive WR stars. Unlike in the $1 \mathrm{D} \sim 5 M_{\odot}$ ejecta model of Woosley et al. (1999), only a small fraction of the ejecta mass is rich in ${ }^{56} \mathrm{Ni}$, contributing around bolometric maximum, while the longer time light curve is powered by a modest mass of ${ }^{56} \mathrm{Ni}$ but tied to a more massive ejecta. In this context, the mass-loss and angular-momentum-loss problem that plagues LGRB/SN progenitors no longer holds. Furthermore, if we argue for the formation of a massive $\mathrm{BH}$, massive WR stars (as massive as models 35OB-35OC in Woosley \& Heger 2006) are suitable for producing an LGRB/SN. This model still requires fastrotating progenitors evolved at low metallicity (Hirschi et al. 2004; Yoon \& Langer 2005; Georgy et al. 2009), but there is no longer a need to reach down to final WR star masses as low as $5 M_{\odot}$. The possibility of a fast-rotating massive WR progenitor for LGRB/SNe means that the proto-magnetar model is not the only viable model. Mazzali et al. (2014) favour the protomagnetar model of LGRB/SNe because their energetics are comparable to the initial energy of a millisecond-period magnetar. This may be fortuitous. For example, standard core-collapse and thermonuclear SNe have a similar explosion energy but their explosion mechanism is entirely different and their progenitors come from distinct stellar populations. Wang et al. (2017) argue that the early peak and late-time slow decline in the light curves support the proto-magnetar model because no other model can match this feature. However, our model shows that there is an alternative. The early peak and late-time slow decline in the light curves may be indicative of a highly ejecta asymmetry, and our model spectra at the photospheric and nebular phases support this.

Spherically symmetric models of LGRB/SN light curves and spectra are characterised by energies up to half the binding energy of a neutron star. By invoking asymmetry, LGRB/SNe may have ejecta energies $\$ 10^{52} \mathrm{erg}$, thus are more compatible with expectations of what can be produced in fast-rotating progenitors. At cosmological redshifts, we tend to detect events in which the bulk of the energy is injected along our direction and then infer a large isotropic luminosity, while we fail to detect those in which the energy is deposited away from our line of sight, even though they may have the same energetics. LGRB/SNe are extremely rare events relative to the ensemble of core-collapse $\mathrm{SNe}$ (see e.g. Podsiadlowski et al. 2004), so the reduced ${ }^{56} \mathrm{Ni}$ mass brought in by considering asphericity would have little impact 

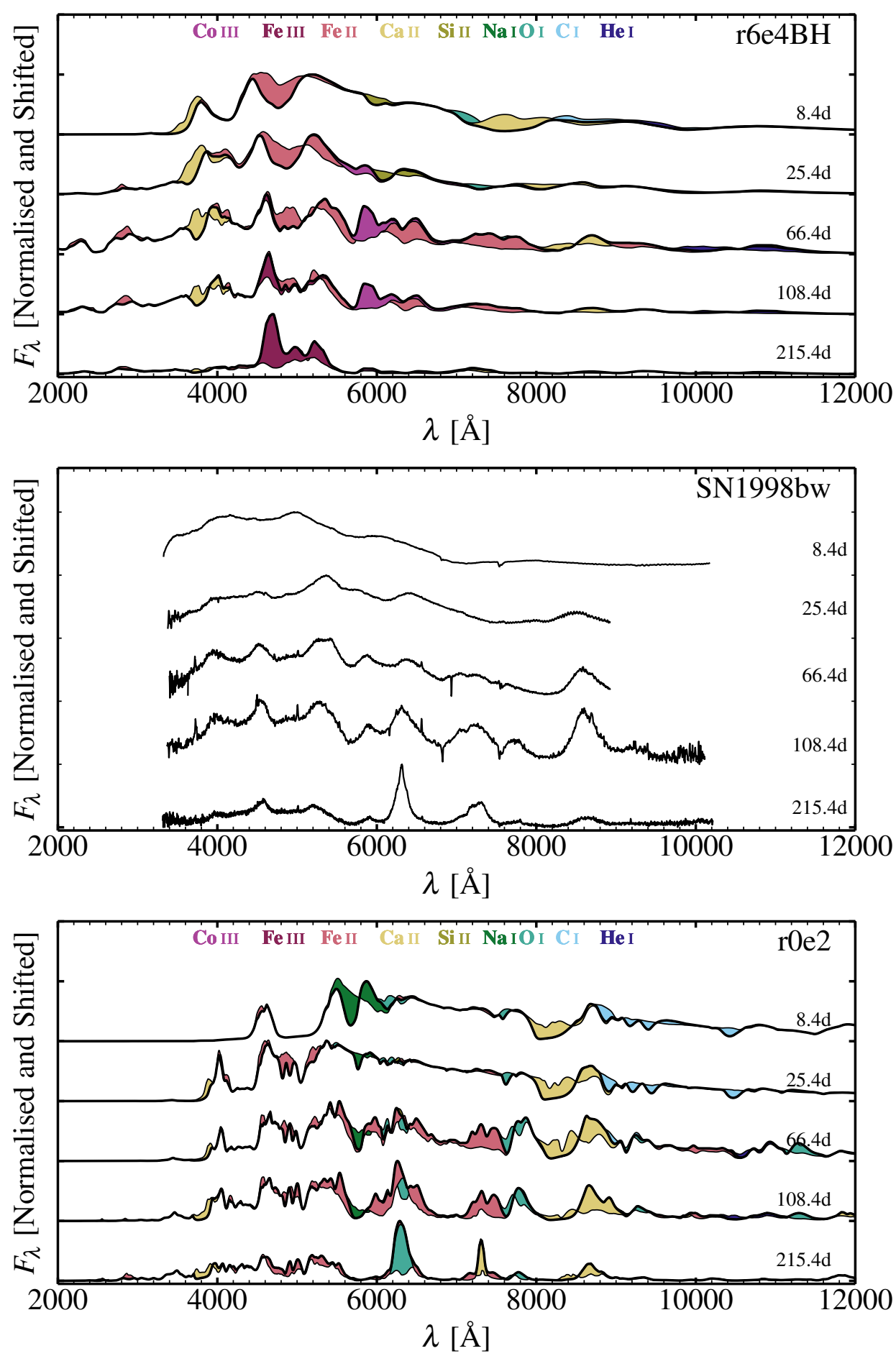

Fig. 5. Multi-epoch spectra of SN 1998bw (centre; corrected for redshift and extinction), and of models r6e4BH (top) and r0e2 (bottom). Times are given since MJD 50929.4 for SN 1998bw and since explosion for the models. We shade the model flux associated with bound-bound transitions of specific ions (although instructive, this procedure only works accurately in the absence of line overlap). Neither model matches the whole evolution of SN 1998bw. However, the lower mass and higher energy model r6e4BH more closely resembles SN 1998bw at early times while the more massive and lower energy model r0e 2 matches better at late times.

on the chemical enrichment of the Universe; the higher mass ejecta would however favour a greater release of $\mathrm{O}$ from lower latitudes.

Even today, the majority of analyses on LGRB/SNe and standard $\mathrm{SNe}$ Ic are based on simplistic arguments for the light curve (Drout et al. 2011; D'Elia et al. 2015; Prentice et al. 2016). Volnova et al. (2017) performed detailed radiationhydrodynamics simulations of LGRB/SN 2013dx, but their modelling is $1 \mathrm{D}$ and they focus on the early-time radiation only. When applied to the asymmetric ejecta of LGRB/SNe, such studies can lead to systematic errors in the determination of ejecta parameters.

The large mass budget inferred for SN 1998bw makes it possible to argue for BH formation. As discussed in Dessart et al. (2012), the final density structure of the $12-16 M_{\odot}$ models of Woosley \& Heger (2006) proposed as LGRB/SN progenitors is 
similar to that of standard non-rotating $15 M_{\odot}$ RSG stars. The latter are expected to produce garden variety SNe II-P, so it is unlikely that the former could produce the extreme properties necessary to make LGRB/SNe, which are also very rare events. On the other hand, the massive WR models 35OB/35OC of Woosley \& Heger (2006), with their massive Fe cores and high compactness, now appear suitable to form a collapsar and an LGRB/SN like SN 1998bw.

Acknowledgements. D.J.H. acknowledges support from NASA theory grant NNX10AC80G, and S.C.Y. acknowledges support from the Korea Astronomy and Space Science Institute under the R\&D programme (Project No. 334820160002) supervised by the Ministry of Science, ICT and Future Planning. This work utilised computing resources of the mesocentre SIGAMM, hosted by the Observatoire de la Côte d'Azur, Nice, France.

\section{References}

Blondin, S., Dessart, L., Hillier, D. J., \& Khokhlov, A. M. 2013, MNRAS, 429, 2127

Bucciantini, N., Quataert, E., Arons, J., Metzger, B. D., \& Thompson, T. A. 2008, MNRAS, 383, L25

Crowther, P. A. 2007, ARA\&A, 45, 177

D’Elia, V., Pian, E., Melandri, A., et al. 2015, A\&A, 577, A116

Dessart, L. 2015, in Wolf-Rayet Stars: Proc. of an International Workshop held in Potsdam, Germany, 1-5 June 2015, eds. W.-R. Hamann, A. Sander, H. Todt (Universitätsverlag Potsdam), 245

Dessart, L., Burrows, A., Livne, E., \& Ott, C. D. 2008, ApJ, 673, L43

Dessart, L., Livne, E., \& Waldman, R. 2010a, MNRAS, 408, 827

Dessart, L., Livne, E., \& Waldman, R. 2010b, MNRAS, 405, 2113

Dessart, L., Hillier, D. J., Livne, E., et al. 2011, MNRAS, 414, 2985

Dessart, L., O’Connor, E., \& Ott, C. D. 2012, ApJ, 754, 76

Dessart, L., Hillier, D. J., Blondin, S., \& Khokhlov, A. 2014a, MNRAS, 439, 3114

Dessart, L., Hillier, D. J., Blondin, S., \& Khokhlov, A. 2014b, MNRAS, 441, 3249

Dessart, L., Hillier, D. J., Woosley, S., et al. 2015, MNRAS, 453, 2189

Dessart, L., Hillier, D. J., Woosley, S., et al. 2016, MNRAS, 458, 1618

Drout, M. R., Soderberg, A. M., Gal-Yam, A., et al. 2011, ApJ, 741, 97

Eldridge, J. J., Izzard, R. G., \& Tout, C. A. 2008, MNRAS, 384, 1109

Ensman, L. M., \& Woosley, S. E. 1988, ApJ, 333, 754

Fruchter, A. S., Levan, A. J., Strolger, L., et al. 2006, Nature, 441, 463

Galama, T. J., Vreeswijk, P. M., van Paradijs, J., et al. 1998, Nature, 395, 670

Georgy, C., Meynet, G., Walder, R., Folini, D., \& Maeder, A. 2009, A\&A, 502, 611

Glebbeek, E., Gaburov, E., de Mink, S. E., Pols, O. R., \& Portegies Zwart, S. F. 2009, A\&A, 497, 255

Groh, J. H., Meynet, G., Georgy, C., \& Ekström, S. 2013, A\&A, 558, A131

Hillier, D. J., \& Dessart, L. 2012, MNRAS, 424, 252

Hirschi, R., Meynet, G., \& Maeder, A. 2004, A\&A, 425, 649
Höflich, P., Wheeler, J. C., \& Wang, L. 1999, ApJ, 521, 179 Iwamoto, K., Mazzali, P. A., Nomoto, K., et al. 1998, Nature, 395, 672 Jerkstrand, A., Fransson, C., \& Kozma, C. 2011, A\&A, 530, A45 Langer, N., Hamann, W.-R., Lennon, M., et al. 1994, A\&A, 290

Le Floc'h, E., Duc, P.-A., Mirabel, I. F., et al. 2003, A\&A, 400, 499

Lisakov, S. M., Dessart, L., Hillier, D. J., Waldman, R., \& Livne, E. 2017, MNRAS, 466, 34

Liu, Y.-Q., Modjaz, M., Bianco, F. B., \& Graur, O. 2016, ApJ, 827, 90 Livne, E. 1993, ApJ, 412, 634

MacFadyen, A. I., \& Woosley, S. E. 1999, ApJ, 524, 262

Maeder, A., \& Meynet, G. 1994, A\&A, 287

Maeda, K., Nakamura, T., Nomoto, K., et al. 2002, ApJ, 565, 405

Maeda, K., Mazzali, P. A., Deng, J., et al. 2003, ApJ, 593, 931

Maeda, K., Nomoto, K., Mazzali, P. A., \& Deng, J. 2006, ApJ, 640, 854

Maeda, K., Kawabata, K., Mazzali, P. A., et al. 2008, Science, 319, 122

Mazzali, P. A., Iwamoto, K., \& Nomoto, K. 2000, ApJ, 545, 407

Mazzali, P. A., McFadyen, A. I., Woosley, S. E., Pian, E., \& Tanaka, M. 2014, MNRAS, 443, 67

McKenzie, E. H., \& Schaefer, B. E. 1999, PASP, 111, 964

Metzger, B. D., Giannios, D., Thompson, T. A., Bucciantini, N., \& Quataert, E. 2011, MNRAS, 413, 2031

Modjaz, M., Kewley, L., Kirshner, R. P., et al. 2008, AJ, 135, 1136

Modjaz, M., Liu, Y. Q., Bianco, F. B., \& Graur, O. 2016, ApJ, 832, 108

Nakamura, T., Mazzali, P. A., Nomoto, K., \& Iwamoto, K. 2001, ApJ, 550, 991

Nieuwenhuijzen, H., \& de Jager, C. 1990, A\&A, 231, 134

Nugis, T., \& Lamers, H. J. G. L. M. 2000, A\&A, 360, 227

Patat, F., Cappellaro, E., Danziger, J., et al. 2001, ApJ, 555, 900

Paxton, B., Bildsten, L., Dotter, A., et al. 2011, ApJS, 192, 3

Paxton, B., Cantiello, M., Arras, P., et al. 2013, ApJS, 208, 4

Paxton, B., Marchant, P., Schwab, J., et al. 2015, ApJS, 220, 15

Podsiadlowski, P., Joss, P. C., \& Hsu, J. J. L. 1992, ApJ, 391, 246

Podsiadlowski, P., Mazzali, P. A., Nomoto, K., Lazzati, D., \& Cappellaro, E. 2004, ApJ, 607, L17

Prentice, S. J., Mazzali, P. A., Pian, E., et al. 2016, MNRAS, 458, 2973

Scalzo, R., Aldering, G., Antilogus, P., et al. 2014, MNRAS, 440, 1498

Sollerman, J., Kozma, C., Fransson, C., et al. 2000, ApJ, 537, L127

Taddia, F., Sollerman, J., Leloudas, G., et al. 2015, A\&A, 574, A60

Toy, V. L., Cenko, S. B., Silverman, J. M., et al. 2016, ApJ, 818, 79

Valenti, S., Benetti, S., Cappellaro, E., et al. 2008, MNRAS, 383, 1485

Vink, J. S., de Koter, A., \& Lamers, H. J. G. L. M. 2001, A\&A, 369, 574

Volnova, A. A., Pruzhinskaya, M. V., Pozanenko, A. S., et al. 2017, MNRAS, 467, 3500

Wang, L. J., Yu, H., Liu, L. D., et al. 2017, ApJ, 837, 128

Wheeler, J. C., Yi, I., Höflich, P., \& Wang, L. 2000, ApJ, 537, 810

Wongwathanarat, A., Mueller, E., \& Janka, H.-T. 2015, A\&A, 577, A48

Woosley, S. E. 1993, ApJ, 405, 273

Woosley, S. E., \& Heger, A. 2006, ApJ, 637, 914

Woosley, S. E., \& Weaver, T. A. 1995, ApJS, 101, 181

Woosley, S. E., Eastman, R. G., \& Schmidt, B. P. 1999, ApJ, 516, 788

Yoon, S.-C. 2015, PASA, 32, 15

Yoon, S.-C., \& Langer, N. 2005, A\&A, 443, 643

Yoon, S.-C., Langer, N., \& Norman, C. 2006, A\&A, 460, 199 


\section{Appendix A: Line identifications in model $\mathrm{rOe} 2$ at multiple epochs}

In this section, we present a montage of spectra for model r0e2 that illustrates the bound-bound contributions from selected ions at four consecutive epochs.
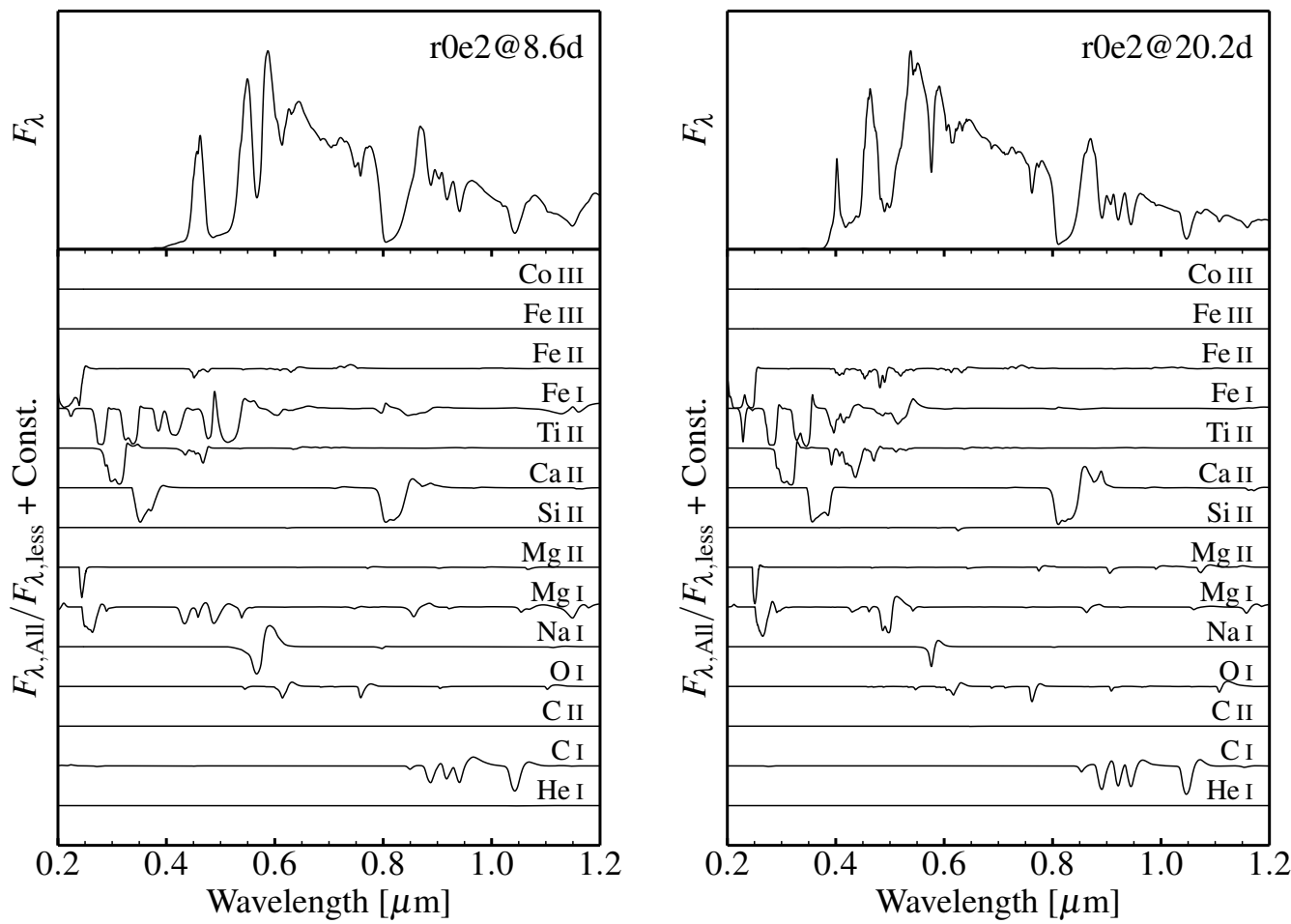

Fig. A.1. Montage of spectra for model r0e2 for post-explosion times of 8.6 (left) and $20.2 \mathrm{~d}($ right $)$. In the top part of each panel, we show the total spectrum, while below, we stack the ratio between the full synthetic spectrum $\left(F_{\lambda, \text { All }}\right)$ and the synthetic spectrum computed by ignoring the bound-bound transitions of a given ion ( $F_{\lambda, \text { less }}$; as indicated by the label on the right).
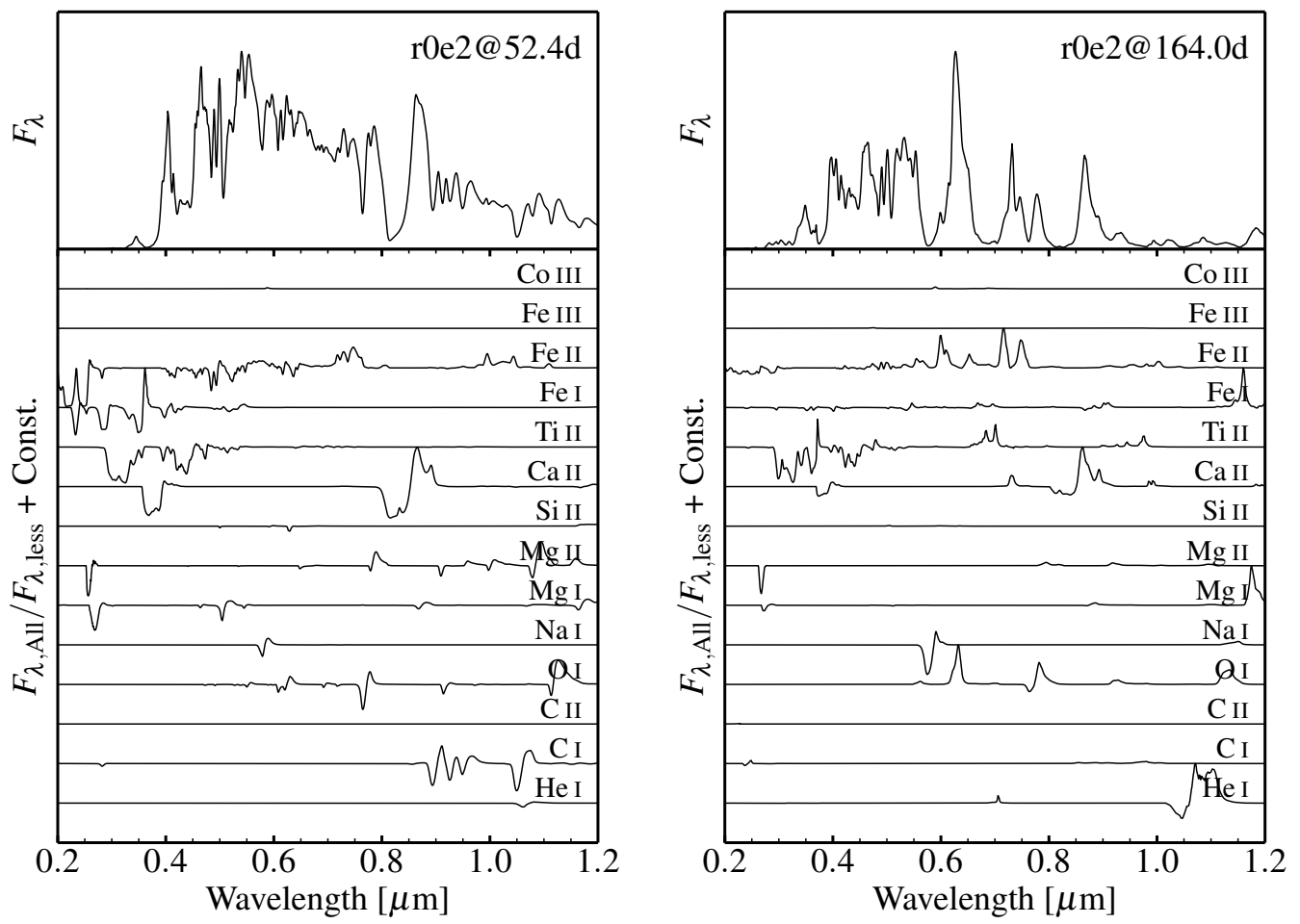

Fig. A.2. Same as Fig. A.1, but now for post-explosion times 52.4 (left) and $164.0 \mathrm{~d}($ right). 


\section{Appendix B: Line identifications in model r6e4BH at multiple epochs}

In this section, we present a montage of spectra for model r6e4BH, which illustrates the bound-bound contributions from selected ions at four consecutive epochs.
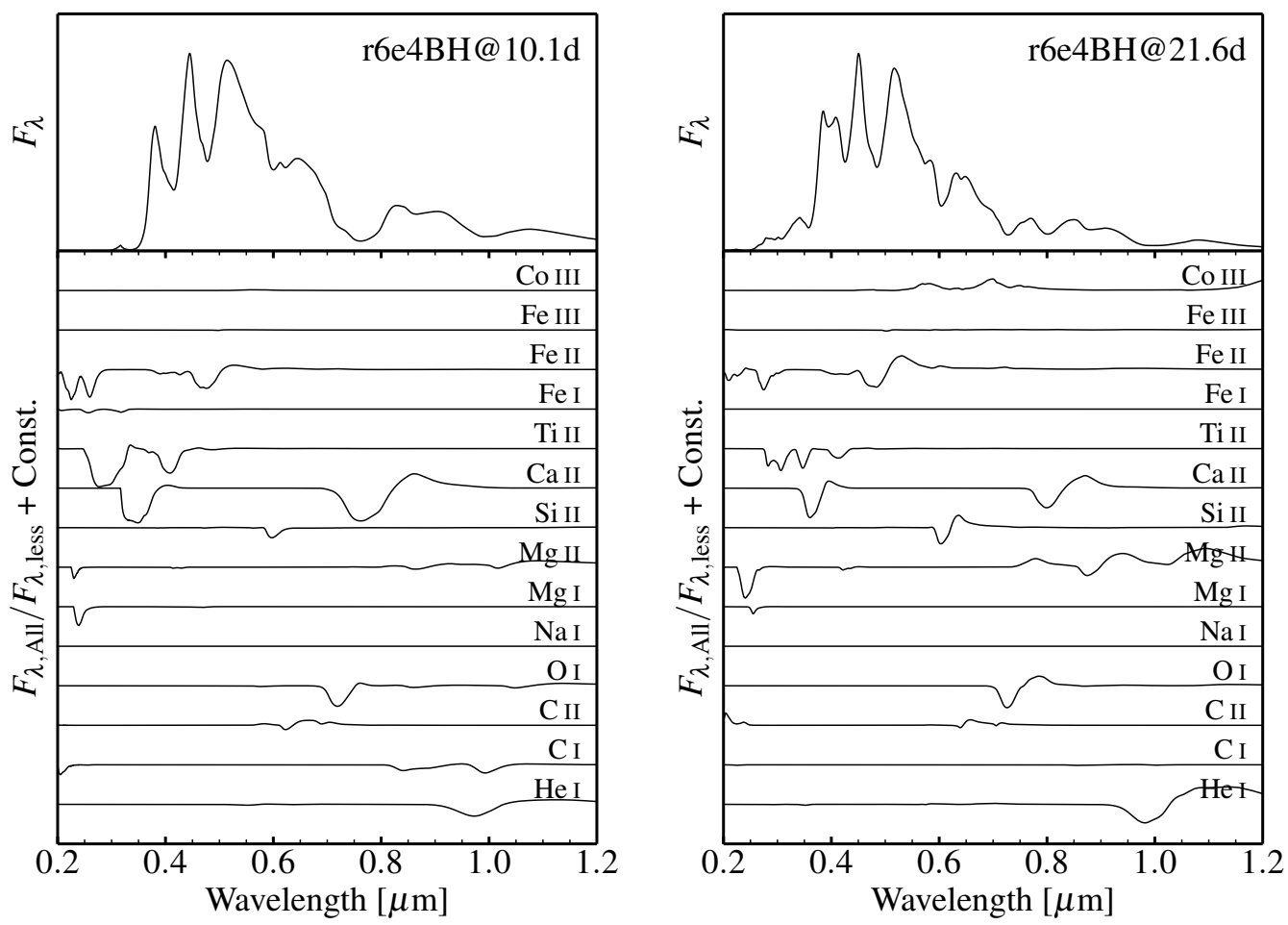

Fig. B.1. Same as Fig. A.1, but now for model r6e4BH at post-explosion times of 10.1 (left) and 21.6d (right).
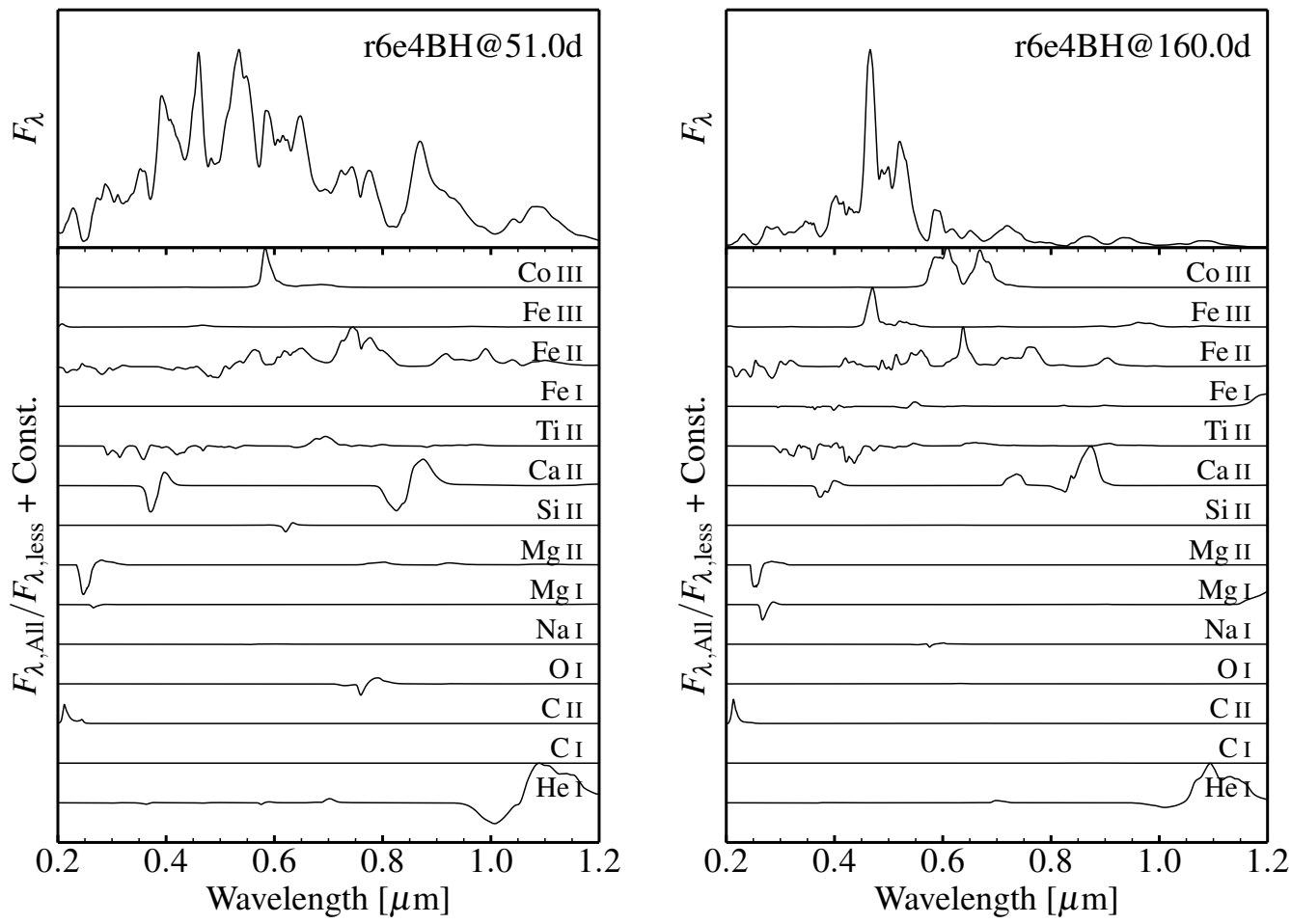

Fig. B.2. Same as Fig. B.1, but now for post-explosion times 51.0 (left) and $160.0 \mathrm{~d}($ right). 